Proyecciones Journal of Mathematics

Vol. 34, No 1, pp. 55-68, March 2015.

Universidad Católica del Norte

Antofagasta - Chile

\title{
Square Sum Labeling of Class of Planar Graphs
}

\author{
Reena Sebastian \\ S. E. S. College, India \\ and \\ K. A. Germina \\ Central University of Kerala, India \\ Received : November 2013. Accepted : February 2015
}

\begin{abstract}
$A(p, q)$ graph $G$ is said to be square sum, if there exists a bijection $f: V(G) \rightarrow\{0,1,2, \ldots, p-1\}$ such that the induced function $f^{*}: E(G) \rightarrow N$ defined by $f^{*}(u v)=(f(u))^{2}+(f(v))^{2}$, for every $u v \in E(G)$ is injective. In this paper we proved that the planar graphs $P l_{m, n}, T B L(n, \alpha, k, \beta)$ and higher order level joined planar grid admits square sum labeling. Also the square sum properties of several classes of graphs with many odd cycles are studied.
\end{abstract}

Key Words : Square sum graphs, $P l_{m, n}, T B L(n, \alpha, k, \beta)$.

\footnotetext{
${ }^{1}$ First author is indebted to the University Grants Commission(UGC) for granting her Teacher Fellowship under UGC's Faculty Development Programme during XI plan. footnote
} 


\section{Introduction}

Unless mentioned otherwise, by a graph we shall mean in this paper a finite, undirected, connected graph without loops or multiple edges. Terms not defined here are used in the sense of Harary [7]. Labeling of a graph $G$ is an assignment of integers either to the vertices or edges or both subject to certain conditions. A dynamic survey to know about the numerous graph labeling method is regularly updated by J.A Gallian [5]. Acharya and Germina defined a square sum labeling of a $(p, q)$-graph $G[1,2]$ as follows.

Definition 1.1. A $(p, q)$-graph $G$ is said to be square sum, if there exists a bijection $f: V(G) \rightarrow\{0,1,2, \ldots, p-1\}$ such that induced function $f^{*}: E(G) \rightarrow N$ defined by $f^{*}(u v)=(f(u))^{2}+(f(v))^{2}$, for every $u v \in E(G)$ is injective.

A connected graph with one cycle is called unicyclic. We proved that all unicyclic graphs are square sum[6]. In this paper we proved that the planar graphs $P l_{m, n}, T B L(n, \alpha, k, \beta)$ and higher order level joined planar grid admits square sum labeling. Also the square sum properties of several classes of graphs with many odd cycles are studied.

Theorem 1.2. [6] Complete graph $K_{n}$ is square sum If and only if $n \leq 5$.

A graph is planar if it has an embedding on plane. $K_{n}, n \leq 4$ are planar. But $K_{n}$ for $n \geq 5$ is non-planar. The complete bipartite graph $K_{m, n}$ is square sum if $m<4$ and for all $n$ [2]. However an attempt is made to find the maximal square sum bipartite planar graph and we show that the planar graph whose definition are based on complete bipartite graphs are square sum. In [9], Babujee defines a class of planar graphs which is obtained from the complete bipartite graph $K_{m, n}, m, n \geq 3$ by removing some edges to make it planar graphs, which is called a bipartite planar class and it is denoted by $P l_{m, n}$.

Definition 1.3. [9] Let $K_{m, n}\left(V_{m}, U_{n}\right)$ be the complete bipartite graph on $V_{m}=\left\{v_{1}, v_{2}, \ldots, v_{m}\right\}$ and $U_{n}=\left\{u_{1}, u_{2}, \ldots, u_{n}\right\}$. The class of graphs $P l_{m, n}(V, E)$ has the vertex set $V_{m} \cup U_{n}$ and the edge set $E=E\left(K_{m, n}\left(V_{m}, U_{n}\right)\right) \backslash\left\{\left(v_{l}, u_{p}\right): 3 \leq l \leq m\right.$ and $\left.2 \leq p \leq n-1\right\}$.

The graph shown in Figure 1 is a bipartite planar graph with $2 m+2 n-4$ edges and $m+n$ vertices. We now describe the embedding used for our 
proofs. Place the vertices $u_{1}, u_{2}, \ldots, u_{n}$ in that order along a horizontal line segment with $u_{1}$ as the left endpoint and $u_{n}$ as the right endpoint as shown in Figure 1. Place the vertices $v_{m}, v_{m-1}, \ldots ., v_{3}, v_{1}$ in that order along a vertical line segment with $v_{m}$ as the top end point and $v_{1}$ as the bottom end point so that this entire line segment is above the horizontal line segment where the vertices $u_{1}$ through $u_{n}$ are placed. Finally place $v_{2}$ below the horizontal line segment so that the vertices $v_{1}, u_{k}, v_{2}, u_{k+1}$ form a face of length 4 for $1 \leq k \leq n-1$. Notice that though we talk about placement along a line segment, no edges other than those mentioned in the definition are to be added.

Here we show that the planar graph $P l_{m, n}$ has square sum labeling.

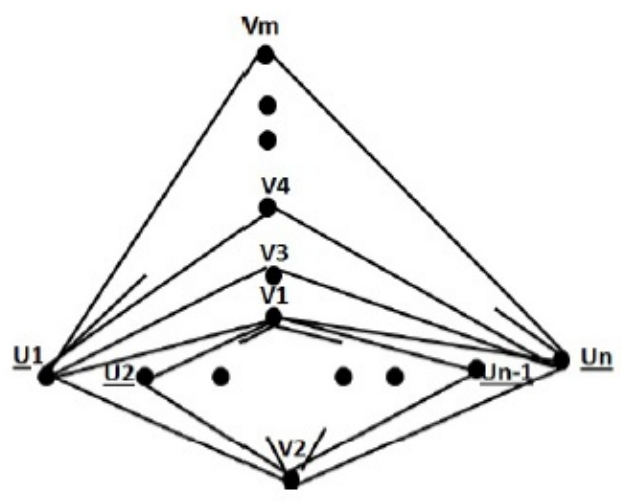

Figure 1: The class $P l_{m, n}$

Theorem 1.4. The graph $P l_{m, n}, m, n, \geq 3$, is a square sum graph.

Proof. Consider the graph $P l_{m, n}(V, E)$, where

$V=\left\{v_{1}, v_{2}, \ldots, v_{m}, u_{1}, u_{2}, \ldots, u_{n}\right\}$. Define the injective function

$f: V\left(P l_{m, n}\right) \rightarrow\{0,1, \ldots, m+n-1\}$ by

$f\left(u_{i}\right)=i-1 ; 2 \leq i \leq n-1$;

$f\left(u_{1}\right)=n-1$;

$f\left(u_{n}\right)=n$;

$f\left(v_{2}\right)=0$ 
$f\left(v_{1}\right)=n+1$

$f\left(v_{i}\right)=f\left(v_{1}\right)+i-2 ; 3 \leq i \leq m$

Clearly $f$ is injective. It is enough to check the labeling of edges with end vertices having labels $(a, a+x)$ and $(a+1, a+y)$ where $a=f\left(u_{1}\right), x>y$. If $a^{2}+(a+x)^{2}=(a+1)^{2}+(a+y)^{2}$, then $k^{2}+2 a k+2 y k=2 a+1$, where $x-y=k$, has no integer solution for any positive integer value of $k$. Hence all the edge labels are distinct.

Definition 1.5. Let $u$ be a vertex of $P_{m} \times P_{n}$ such that $\operatorname{deg}(u)=2$. Introduce an edge between every pair of distinct vertices $v, w$ with $\operatorname{deg}(v)$, $\operatorname{deg}(w) \neq 4$, if $d(u, v)=d(u, w)$, where $d(u, v)$ is the distance between $u$ and $v$. The graph so obtained is defined as the level joined planar grids and it is denoted by $L J_{m, n}$.

An example of $L J_{4,5}$ is illustrated in figure 2.

Theorem 1.6. The graph $L J_{m, n}$ is square sum for all $m, n \geq 2$.

Proof. Denote the vertex at the $i^{\text {th }}$ row and $j^{\text {th }}$ column of $P_{m} \times P_{n}$ as $v_{i, j}$. Without loss of generality we can assume $m \leq n$. Construct the graph $L J_{m, n}$ as illustrated in Figure 2. Let $V$ be the vertex set of $L J_{m, n}$ with $p$ vertices. Define a function $f: V \rightarrow\{0,1,2, \ldots, p-1\}$ such that

$$
\left\{\begin{array}{l}
f\left(v_{1, j}\right)=\frac{j(j+1)-2}{2} ; 1 \leq j \leq m \\
f\left(v_{1, j}\right)=\frac{\left(m+2 m j-m^{2}\right)-2}{2} ; m<j \leq n \\
f\left(v_{i, j}\right)=f\left(v_{i-1, j+1}\right)-1 ; 2 \leq i \leq m, 1 \leq j \leq n+1-i \\
f\left(v_{i, j}\right)=f\left(v_{i-1, j}\right)+(m+n+1-j-i) ; 2 \leq i \leq m, n+1-i<j \leq n
\end{array}\right.
$$

Here $f$ is injective. With the above labeling no two of the edge labels are same as the edge labels can be arranged in an increasing order. Hence $f$ is square sum labeling of $L J_{m, n}$ for all $m, n \geq 2$.

For $\mathrm{m}=1, \mathrm{n}=2$ we get $P_{1} \times P_{2} \equiv P_{2}$ which is square sum. A square sum labeling of level joined planar grid $L J_{4,5}$ using theorem 1.6 is exhibited in Figure 2. 


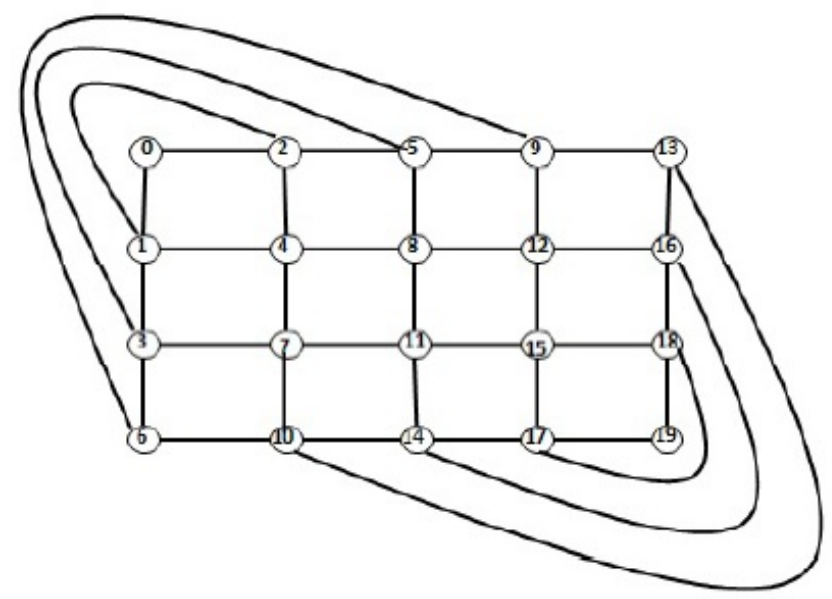

Figure 2 : Square sum labeling of $L J_{4,5}$

Theorem above motivate us to construct a higher order square sum graph. Let $v_{i, j}^{x}$ denote the vertex $v_{i, j}$ in the $x^{t h}$ copy of $L J_{m, n}$. For any integer $t>1$, construct a graph by joining the vertex $v_{m-1, n}^{x}$ to the vertices $v_{1,1}^{x+1}, v_{1,2}^{x+1}, v_{2,1}^{x+1} ; 1 \leq x<t$ and denote the resulting graph as $L J_{m, n}^{t}$ (see Figure 3). The following result is the general form of Theorem 1.6.

Theorem 1.7. The graph $L J_{m, n}^{t}$ is square sum for all $m, n \geq 2$ and $t \geq 1$.

Proof. Denote the vertex of the graph $L J_{m, n}^{t}$ as illustrated. Here $\left|V\left(L J_{m, n}^{t}\right)\right|=m n t$. That is $p=m n t$.

Define a function $f: V\left(L J_{m, n}^{t}\right) \rightarrow\{0,1,2, \ldots, m n t-1\}$ for $1 \leq x \leq t$, by

$$
\left\{\begin{array}{l}
f\left(v_{1, j}^{x}\right)=\frac{j(j+1)-2}{2}+m n(x-1) ; 1 \leq j \leq m \\
f\left(v_{1, j}^{x}\right)=\frac{\left(m+2 m j-m^{2}\right)-2}{2}+m n(x-1) ; m \leq j \leq n \\
f\left(v_{i, j}^{x}\right)=f\left(v_{i-1, j+1}^{x}\right)-1 ; 2 \leq i \leq m, 1 \leq j \leq n+1-i \\
f\left(v_{i, j}^{x}\right)=f\left(v_{i-1, j}^{x}\right)+(m+n+1-j-i) ; 2 \leq i \leq m, n+1-i<j \leq n
\end{array}\right.
$$

Here $f$ is injective. With the above defined vertex labeling the edge labels can be arranged in an increasing order. Hence $f$ so defined is a square sum labeling of graph $L J_{m, n}^{t}$ for all $m, n \geq 2$ and $t \geq 1$. 
For example, a square sum labeling of $L J_{m, n}^{t}$ when $m=4, n=5, t=3$ using theorem 1.7 is illustrated in Figure 3.

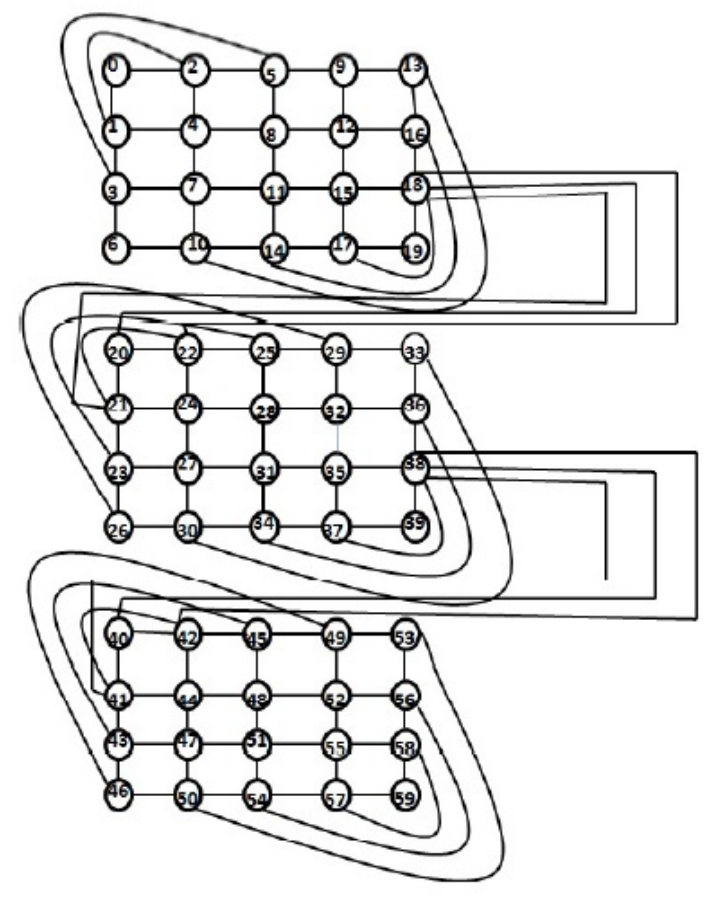

Figure 3 : Square sum labeling of $L J_{4,5}^{3}$

For $n \geq 2$ let $L_{n}$ be the Cartesian product $P_{n} \times P_{2}$ of a path on $n$ vertices with a path on two vertices. In [2] it is proved that $L_{n}$ is square sum for $n \geq 2$.

Let $S=\{\uparrow, \downarrow\}$ be the symbol representing the position of the block (Figure $4)$.
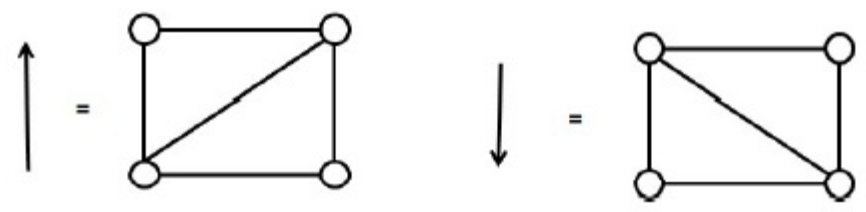

Figure 4 : 
Let $\alpha$ be a sequence of $n$ symbols of $S$, i.e, $\alpha \in S^{n}$. We will construct a graph by tiling $n$-blocks side by side, with their positions indicated by $\alpha$. We will denote the resulting graph by $T B(\alpha)$ and refer to it as a triangular belt. For simplicity we denote $(\uparrow, \uparrow, \uparrow, \ldots, \uparrow)$ by $\uparrow^{n}$ and $(\downarrow, \downarrow, \ldots, \downarrow)$ by $\downarrow^{n}$. Example 1:


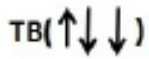

$\mathrm{TB}(\uparrow \downarrow \downarrow \uparrow)$

$\mathrm{TB}(\uparrow \downarrow \uparrow \downarrow)$

$\mathrm{TB}(\uparrow \uparrow \uparrow \uparrow)$

Figure 5 :

Theorem 1.8. For any $\alpha \in S^{n}, n>1$, the triangular belt $T B(\alpha)$ is square sum.

Proof. The following algorithm indicates a labeling pattern. Label the top vertices of belt by numbers $\{0,2, \ldots, 2 n\}$ from left to right respectively. Then assign the bottom vertices of the belt from left to right by all odd numbers $\{1,3,5, \ldots, 2 n+1\}$. With the above defined labeling pattern the edge labels can be arranged in increasing order. Figure 6 illustrates the algorithm for $T B(\alpha), T B(\beta), T B(\gamma)$ and $T B(\delta)$ respectively in example 1 . 


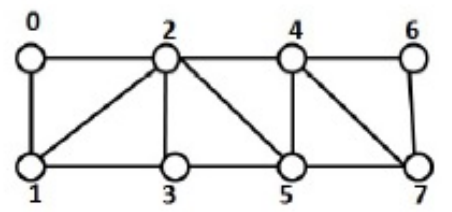

TB( $\downarrow \downarrow)$

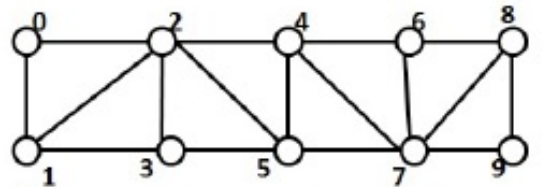

$\mathrm{TB}(\uparrow \downarrow \downarrow \uparrow)$

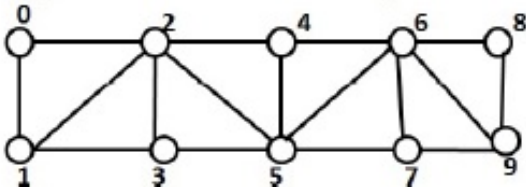

$\mathrm{TB}(\uparrow \downarrow \uparrow \downarrow)$

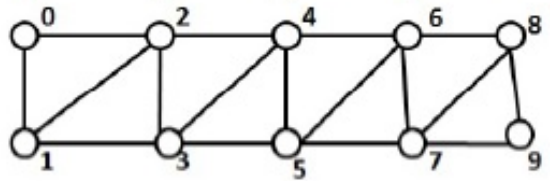

$\mathrm{TB}(\uparrow \uparrow \uparrow \uparrow)$

Figure 6 :

Corollary 1.9. The $2^{\text {nd }}$ power of $P_{n}$ is square sum since it is isomorphic to $T B(\uparrow, \uparrow, \uparrow, \ldots, \uparrow)$ or $T B(\downarrow, \downarrow, \downarrow, \ldots, \downarrow)$.

We now consider a class of planar graphs that are formed by amalgamation of triangular belts. For each $n>1$ and $\alpha \in S^{n}$, we take the triangular belt $T B(\alpha)$ and triangular belt $T B(\beta)$ where $k>0, k$ blocks with last block is $\uparrow$. We rotate $T B(\beta)$ by 90 degrees counter clockwise and amalgamate last block with the first block of $T B(\alpha)$ by sharing an edge. The resulting graph is denoted by $T B L(n, \alpha, k, \beta)$. The graph $T B L(n, \alpha, k, \beta)$ has $2(n+k+1)$ vertices with $V(T B L(n, \alpha, k, \beta)=$ $\left\{x_{1,1}, x_{1,2}, \ldots, x_{1, n+1}, x_{2,1}, x_{2,2}, x_{2, n+1}, y_{3,1}, y_{3,2}, \ldots, y_{3, k}, y_{4,1}, y_{4,2}, \ldots, y_{4, k}\right\}$ (figure 7).

Theorem 1.10. The graph $T B L(n, \alpha, k, \beta)$ is square sum for all $\alpha$ in $S^{n}$ and $\beta$ in $S^{k}$ with last block is being $\uparrow$ for all $k>0$. 
Proof. Define $f: V(T B L(n, \alpha, k, \beta)) \rightarrow\{0,1, \ldots,, 2 n+2 k+1\}$ as follows.

$$
\begin{aligned}
& x_{1,1}=2 n \\
& x_{1, j}=2 n-(2 j-2) ; 2 \leq j \leq n+1 \\
& x_{2,1}=2 n+1 \\
& x_{2, j}=2 n+1-(2 j-2) ; 2 \leq j \leq n+1 \\
& y_{3,1}=2(n+k)+1 \\
& y_{3, j}=2(n+k)+1-(2 j-2) ; 2 \leq j \leq k \\
& y_{4,1}=2(n+k) \\
& y_{4, j}=2(n+k)-(2 j-2) ; 2 \leq j \leq k .
\end{aligned}
$$

Clearly $f$ is injective. Here the only possible case, where there may be edges having same label is edges with end vertices $(a, a+3)$ and $(a+1, a+2)$, where $a=2 n-1$. If $a^{2}+(a+3)^{2}=(a+1)^{2}+(a+2)^{2}$, we get $9=5$, is not possible. Hence all the edge labels are distinct.

Example 1.11. Figure 7 illustrates $k=1,3$ for Theorem 1.10

When $\mathrm{K}=1$

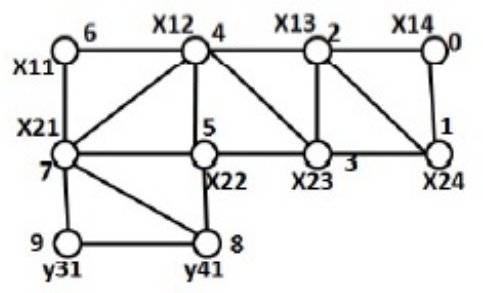

TBL(3, $\uparrow \downarrow \downarrow, 1)$

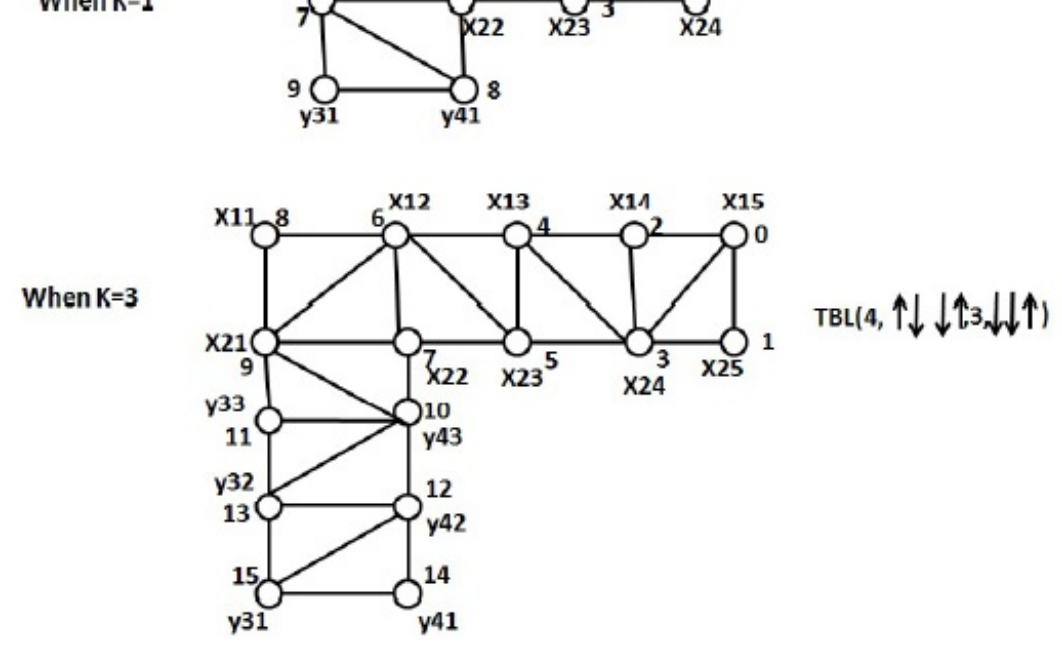

Figure 7 : 
Theorem 1.12. For $k \geq 1$, the planar graph $\left(P_{2} \cup k K_{1}\right)+N_{2}$ is square sum.

Proof. Let the vertex set of $\left(P_{2} \cup k K_{1}\right)$ be $\left\{z_{1}, z_{2}, x_{1}, x_{2}, \ldots, x_{k}\right\}$ and $V\left(N_{2}\right)=\left\{y_{1}, y_{2}\right\}$.

$\left|V\left(P_{2} \cup k K_{1}\right)+N_{2}\right|=k+4$.

Define a labeling $f: V\left(P_{2} \cup k K_{1}\right)+N_{2} \rightarrow\{0,1,2, \ldots, k+3\}$ by $f\left(z_{1}\right)=$ $0, f\left(y_{1}\right)=1, f\left(y_{2}\right)=2, f\left(z_{2}\right)=3$

$f\left(x_{i}\right)=f\left(z_{2}\right)+i ; 1 \leq i \leq k$

Clearly $f$ is injective. It is enough to check the labeling of edges with end vertices having labels $(a, a+x)$ and $(a+1, a+y)$ where $a=f\left(y_{1}\right), x>y$. If $a^{2}+(a+x)^{2}=(a+1)^{2}+(a+y)^{2}$, then $k^{2}+2 a k+2 y k=2 a+1$, where $x-y=k$, has no integer solution for any positive integer value of $k$. Hence all the edge labels are distinct.

Example 1.13. Square sum labeling of $\left(P_{2} \cup 3 K_{1}\right)+N_{2}$

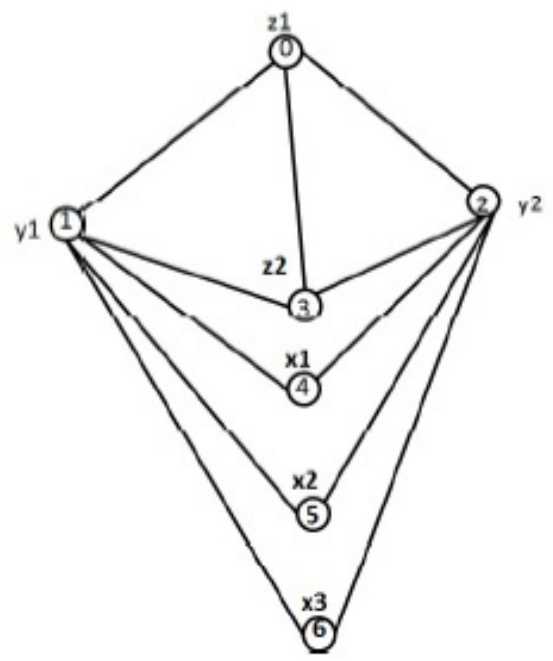

Figure 8 : 


\section{Square sum graph with many odd cycles}

We would like to consider the graphs with many cycles. Let $P_{n}(+) N_{m}$ be the graph with $p=n+m$ and $q=n+2 m-1$.

$V\left(P_{n}(+) N_{m}\right)=\left\{v_{1}, v_{2}, \ldots, v_{n}, y_{1}, y_{2}, \ldots, y_{m}\right\}$ where $V\left(P_{n}\right)=\left\{v_{1}, v_{2} \ldots, v_{n}\right\}$ and $V\left(N_{m}\right)=\left\{y_{1}, y_{2}, \ldots, y_{m}\right\}$ $E\left(P_{n}(+) N_{m}\right)=E\left(P_{n}\right) \cup\left\{\left(v_{1}, y_{1}\right)\left(v_{1}, y_{2}\right), \ldots,\left(v_{1}, y_{m}\right)\left(v_{n}, y_{1}\right)\left(v_{n}, y_{2}\right), \ldots\right.$, $\left.\left(v_{n}, y_{m}\right)\right\}$.

The graph has $m$-cycles of length $n+1$ and many 4-cycles.

Theorem 2.1. $P_{n}(+) N_{m}$ is square sum for all $n, m \geq 1$.

Proof. Define $f: V\left(\operatorname{Pn}(+) N_{m}\right) \rightarrow\{0,1, \ldots, m+n-1\}$ as follows.

Case 1. When $n$ is odd.

$f\left(v_{\left\lceil\frac{n}{2}\right\rceil}\right)=0$

Start from $v_{\left\lceil\frac{n}{2}\right\rceil}$ apply BFS algorithm and label the vertices as $0,1, \ldots, n+$ $m-1$ in increasing order, the order in which they are visited. It is enough to check the labeling of edges with end vertices having labels $(a, a+x)$ and $(a+1, a+y)$ where $a=f\left(v_{1}\right), x>y$. If $a^{2}+(a+x)^{2}=(a+1)^{2}+(a+y)^{2}$, then $k^{2}+2 a k+2 y k=2 a+1$, where $x-y=k$, has no integer solution for any positive integer value of $k$. Hence all the edge labels are distinct.

Case2. When $n$ is even.

Define $f\left(v_{\frac{n}{2}+1}\right)=0$. Start from $v_{\frac{n}{2}+1}$, apply BFS algorithm and label the vertices as $0,1, \ldots, n+m-1$, in increasing order, the order in which they are visited. It is enough to check the labeling of edges with end vertices having labels $(a, a+x)$ and $(a+1, a+y)$ where $a=f\left(v_{1}\right), x>y$. If $a^{2}+(a+x)^{2}=(a+1)^{2}+(a+y)^{2}$, then $k^{2}+2 a k+2 y k=2 a+1$, where $x-y=k$, has no integer solution for any positive integer value of $k$. Hence all the edge labels are distinct. 

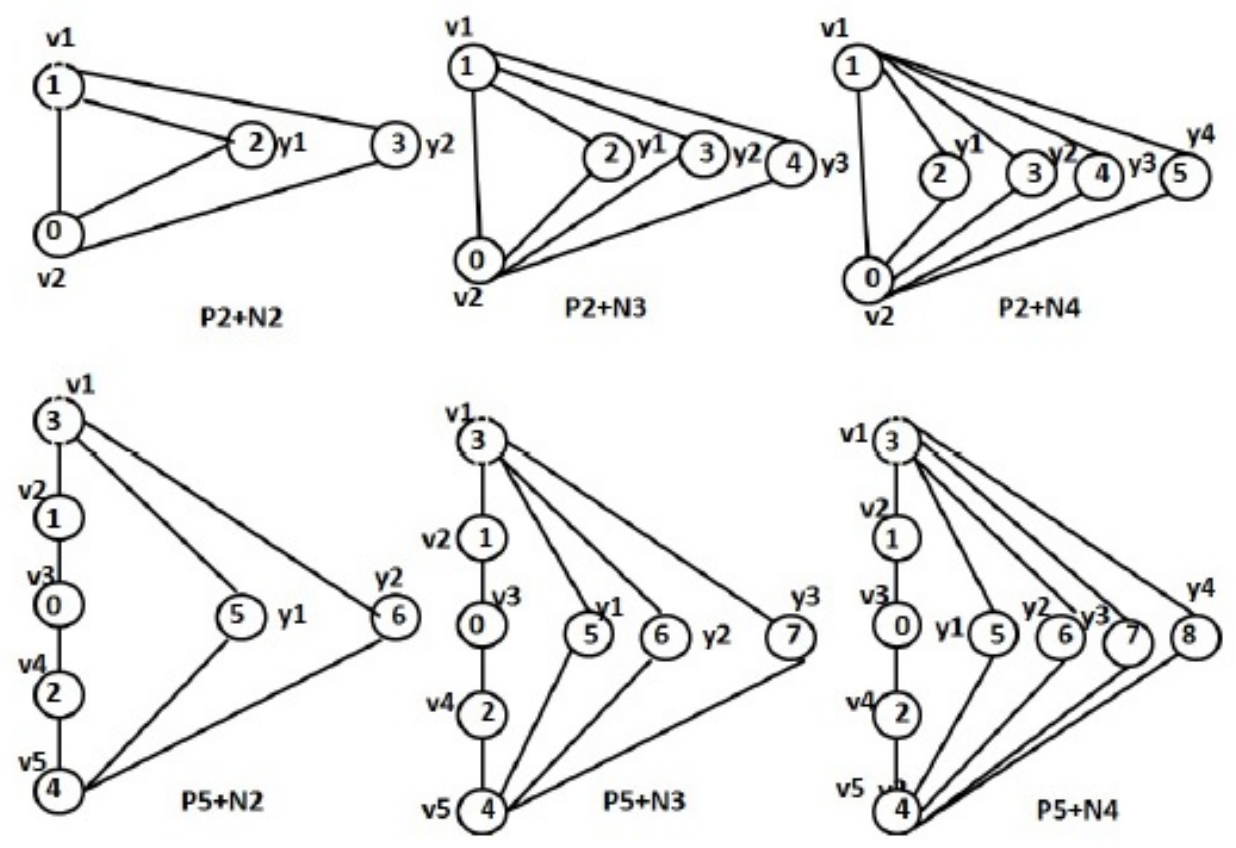

Figure 9 : Square sum labeling of $P_{2}(+) N_{m}$ and $P_{5}(+) N_{m}$, for $m=2,3$ and 4

For integers $m, n \geq 0$, we consider the graph $J(m, n)$ with vertex set $V(J(m, n))=\{u, v, x, y\} \cup\left\{x_{1}, x_{2}, \ldots, x_{m}\right\} \cup\left\{y_{1}, y_{2}, \ldots, y_{n}\right\}$ and edge set $E(J(m, n))=\{(u, x),(u, v),(u, y),(v, x),(v, y)\} \cup\left\{\left(x_{i}, x\right): i=1,2, \ldots, m\right\} \cup$ $\left\{\left(y_{i}, y\right): i=1,2, \ldots, n\right\}$.

We will refer to $J(m, n)$ as a Jellyfish graph.

Theorem 2.2. The Jellyfish graph $J(m, n)$ is square sum for all $m, n \geq 0$. 
Proof. We define a labeling $f: V(J(m, n)) \rightarrow\{0,1, \ldots, m+n+3\}$ as follows.

$f\left(x_{i}\right)=i ; 1 \leq i \leq m$

$f(u)=m+1, f(x)=0$

$f(v)=m+2 ; f(y)=m+3$

$f\left(y_{i}\right)=m+3+i ; 1 \leq i \leq n$.

With the above defined labeling we can arrange the edge labels in an increasing order.

Example 2.3. Square sum labeling of Jellyfish graph $J(3,4)$ is shown in Figure 10.

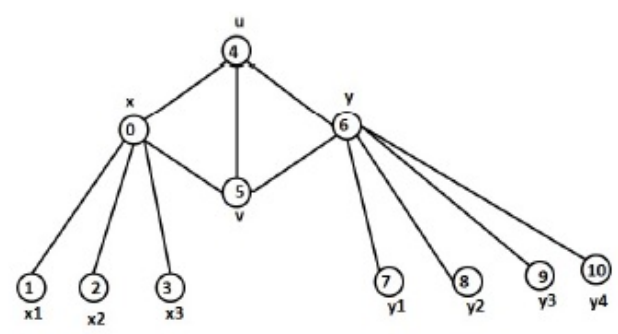

Figure 10 :

\section{References}

[1] B. D. Acharya, Personal Communication, September, (2011).

[2] Ajitha V, Studies in Graph Theory-Labeling of Graphs, Ph. D. Thesis, Kannur Univeristy, Kannur, (2007).

[3] D. M. Burton, Elementary number theory, Second Edition, Wm. C. Brown Company publishers, (1980).

[4] Beineke, L. W. and Hegde, S. M. Strongly Multiplicative graphs, Discuss. Math. Graph Theory, 21, pp. 63-75, (2001). 
[5] J. A. Gallian, A dynamic survey of graph labrling, The Electronic Journal of Combinatorics (DS6), (2005).

[6] Germina K. A. and Reena Sebastian, On square sum Graphs, communicated.

[7] F. Harary, Graph Theory, Addison-Wesley Pub. Comp., Reading, Massachusetts, (1969).

[8] Ersazig and A. Rosa, Magic valuation of finite graphs, Canad. Math. Bull., 13, pp. 451-461, (1970).

[9] K. Ramanjaneyulu, V. Ch. Venkaiah and Kishore Kothapalli, Antimagic labeling of class of planar graphs, Australian Journal of Combinatorics, 41, pp. 283-290, (2008).

[10] J. Baskar Babujee,planar graphs with maximum edge antimagic property, The Mathematics Education, 37 (4), pp. 194-198, (2003).

\section{Reena Sebastian}

Department of Mathematics,

S. E. S. College,

Sreekandapuram,

India

e-mail: reeshijo@gmail.com

and

\section{K. A. Germina}

School of Mathematical and Physical Sciences, Central University of Kerala,

Kasargode,

India

e-mail: srgerminaka@gmail.com 